\title{
Gender Dysphoria in a Mother and Son: A Report of Two Cases Referred to Legal Medicine Office of Iran
}

\author{
Nowroozi A ${ }^{1}$, Borji $\mathrm{E}^{2}$ and Saberi $\mathrm{SM}^{2 *}$ \\ ${ }^{1}$ Iranian National Center for Addiction Studies (INCAS), Tehran University of Medical Sciences \\ (TUMS), Tehran, Iran \\ ${ }^{2}$ Psychiatrist, Legal Medicine Research Center, Legal Medicine Orgasnization, Tehran, Iran
}

*Corresponding author: Saberi SM, Psychiatrist, Legal Medicine Research Center, Legal Medicine Orgasnization, Tehran, Iran, Email: saberi_md@yahoo.com

\section{Case Report \\ Volume 4 Issue 1}

Received Date: February 13, 2020

Published Date: March 19, 2020

DOI: $10.23880 / \mathrm{mhrij}-16000138$

\section{Abstract}

Two cases of gender dysphoria are presented in this article, a mother and her son who had requested legal permission for sex reassignment surgery. The cases were a 16-year-old boy and her 38-year-old biological mother who were referred to the department of forensic psychiatry in Legal Medicine Organization of Iran. They were referred to special clinics for psychiatric evaluations and finally the diagnosis "gender dysphoria" was confirmed. They were not met any criteria for other mental disorders and had not any physical abnormality. Their sex chromosomes were consistent with their physical identities. The results support the possibility of familial aggregation or a shared state of gender identity problems because of a pathologic impressibility and imitation and/or a genetic predisposition of gender dysphoria.

Keywords: Gender Dysphoria; Sex Reassignment; Shared Identity Problem; Genetic Predisposition

\section{Introduction}

Gender dysphoria (GD) is a general descriptive term which refers to people's emotional and cognitive dysphoria regarding their assigned or natal sex. In DSM-5, GD defined as a marked incongruence between one's experienced/ expressed gender and assigned gender that results to clinically significant distress or impairment in one or more areas of functioning [1]. The diagnosis has been replaced gender identity disorder (GID) of DSM-IV-TR [2]. A recent review article reported prevalence of transsexualism among adult natal males and adult natal females as 1 in 14705 and 1 in 38461, respectively [3]. These figures are mainly based on number of clients who refer to specialized clinics and seek hormone therapy or sex reassignment surgery; although population-based studies show that they might underestimate the true prevalence of GS.

Gender ratios of GD clients who refer to specialized clinics are different in age groups. Among natal boys, GD is diagnosed 2 to 4.5 times higher than natal girls [1]. Although girls show more extreme cross-gender behaviors than boys based on parents reports, higher rates of GD diagnosis among assigned boys might explained by lower tolerance of crossgender behaviors of boys than girls by peers and parents [4]. Among adolescents gender ratio of GD diagnosis among boys and girls is almost comparable, although it is slightly higher among boys [5]. Gender ratio of GD diagnosis among adult assigned males is 1.5 to 6 times higher than assigned females [1], although in some countries such as Taiwan [6], Japan and Polanad higher prevalence of GD reported among natal female adults [1].

GD is a complex problem which influenced by interaction of multiple genetic and environmental factors [7]. Family and twin studies investigated the role of genetic factors in development of gender identity and GD $[8,9]$. In one study recalled gender dysphoria among adult twins were investigated. The heritability of GD estimated in a range from 0.5 to 0.57 among females and from 0.37 to 0.4 among males 


\section{Mental Health \& Human Resilience International Journal}

[10]. A review of GID twins' case reports showed 39.1\% concordance rate among monozygotic male and female twins, while none of 21 same-sex dizygotic twins were concordant for GID [8]. Genetic influences of typical behaviors for gender during childhood, sexual attraction and adult gender identity among a sample of British twins were 31\%, 25\% and 11\%, respectively. In another twin study from Sweden, $25-47 \%$ of participants' differences in masculinity-feminity scale could be explained by genetic differences [9]. One study reported 12 pairs of GID patients among identified cases of GID and their probands and showed that probability of GID among siblings of male to female is 4.48 times higher than female to male patients. The probability of GID among brothers was 3.88 times higher than sisters [11].

The aim of this article is to report two cases of gender dysphoria, a 16 years old natal boy (case \#1) and his 38 years old mother (case \#2), requesting legal authorization for sex reassignment surgery (SRS) from Psychiatric department of Legal Medicine Organization (LMO). In Iran, anyone with gender dysphoria has to be legally allowed to change his/her body to the opposite sex by surgery. The patients' data were extracted from their files in LMO consisting of the reports of psychiatrists of the psychiatric council and their previous history of psychiatric and psychotherapeutic care in other settings. They signed a letter of informed consent that the information could be used for research and educational purposes.

\section{Materials and Methods}

\section{Case Reports}

Case 1: M., a 16-year-old, an assigned male at birth, who studies at grade 11 came to Psychiatric department of Tehran Legal Medicine. His chief complaint was "What I am is not consistent with my body". The client was first child of the family and had a brother. He stated that he has had such feeling since he has been 5-6 years old. He would prefer to play often the stereotyped "girlish" games and dress like a girl when he was 5 or 6 years old. He preferred to play with girls and quickly developed a liking to stereotypical girlish games like doll games, playing by one leg and guest game (special girlish plays in Iran). He grew up in a traditional family that did not know about the nature of gender problems, and he had to suffer constant humiliation and feeling of inferiority, especially by his classmates at school. Although his mother had understood his feelings, his father felt ashamed of his appearance and behavior. He told to the psychiatrist that he cannot justify himself and explain the problem to his family members. In these cases, the cultural impact on understanding the issue is undeniable.
He said that he has learned about his problem recently and realized that he could request for SRS. He has thought that he is homosexual at first, but he has suppressed this feeling, since he has believed that "it is a great sin". His parents had seen his girlish behaviors since childhood. He experienced puberty while he was 13 and since then he has had sexual desire to men. In fact, he had never felt any real attraction toward the female sex. During early adolescence; he was attracted to one of his male classmates and has felt disappointment while he has found out that he has a girlfriend. After several years and during the course of therapeutic interventions in specialized psychiatric settings, his father accepted his son's problem. His father emphasized that his son was interested to dance like girls (notice that in Iranian culture boys do not usually dance or behave like girls). He added that his son has been interested to girlish games, to put on girls clothes, and interested to girls sports since early childhood. He reported that his son had been lifting his eyebrows, (it is not common in Iranian culture) but he had not been concerned with that before. He knew the nature of gender dysphoria and that he would not be able to get pregnant after sexual reassignment surgery even in the best possible conditions. The psychiatrist spent more time to explain the good and bad consequences of the surgery and to appreciate the nature and quality of sexual relationship that he may have in the future.

He reported that he likes to putwomen's dress, participate in female gatherings, having permission to make up in home and practicing dance. He was interested to designing women's dresses, female hair styling, housekeeping tasks and fine artistic works. During treatment sessions he started to put girlish clothes regularly and was very satisfied with that. He reported that he is more relaxed in women's dress and people's look does not bother him. He stated that his family accepted him as a girl and he decided to make SRS as soon as possible. His intention for SRS was "to resolve the conflict between his mind and his body" since he feel that "I am a prisoner in a wrong body". Mental status examination revealed dysphoric mood, helplessness, over-valued ideas about her sexual identity and mild anxiety symptoms. During first session, his father used to try to describe his child as a normal boy, although during treatment sessions he had stated that he has paid more attention to his child behaviors recently, and realized that he has "girlish morale and characteristics".

The results of karyotype and sexual hormone tests were in normal range and consistent with his assigned gender as a male. Patient showed high scores in masculinity-femininity (Mf) scale of Minnesota Multiphasic Personality Inventory (MMPI) implying lack of traditional masculine role and interests. 


\section{Mental Health \& Human Resilience International Journal}

He attended in the Psychiatric Council of Legal Medicine Office, and was examined by a group of expert psychiatrists. After a long discussion, they agreed with the sex reassignment surgery.

Case 2: Ms. F, a 38-year-old, an assigned female at birth, mother of case 1, was referred to Psychiatric department of LMO. Her complaint was: "I am not a woman. I am a complete man. I feel I am trapped in a wrong body". She was born in a village in western provinces of Iran. She was a housewife, $7^{\text {th }}$ child of a low socioeconomic family and had completed 5 years of education. She had lost her mother when she was 7 . She was married twice and reported has been mandated to marriage both times. She stated that she had not perceived herself as a woman at all and had never put make up so far. She had chosen a male name for her and reacts to that name. She reported a strong desire to be a male from her childhood. She would prefer to dress like a boy, and play often the known "boyish" games other boys. Her family has been forcing her to put on girl clothes but she hated that. After puberty, she has had sexual attraction towards same-sex and has had sexual experiences with other girls. She said that her family had mandated her to marry with a young man when she was 16 , but she ran away from her husband home after 1 week. The family of her first husband has agreed to divorce, because she had not accepted to come back. After divorce, she left her birthplace and came to Tehran with one of her brothers to find a job at 17 years old. She worked in small factories for a while. She reported bitter experiences to have an independent life as a woman because of financial constraints and need to social support, in Tehran. At the same time, she has regularly practiced karate in a female-specific club and has been attracted to her female coach. She stated that she used to buy flowers for her coach every day and after a while she told her that "I love you like a man and want to be with you." The karate coach has said that I am going to marry with a normal man and this made her disappointed. She reported that she began to smoke cigarette when she heard a negative answer from her coach.

She had to marry again, and she said to her second husband that "I will live with you as a friend." She knew that her sexual orientation has always been toward males, but she used to think that her sense of masculinity would decrease after marriage. She was in severe distress all the time and finally attempted suicide thorough hanging herself, but her husband succeeded to save her life.

She has been very reluctant to do housekeeping tasks at home, but was not interested to have sex with her husband, and this led to severe marital conflicts. Their sexual relationships were completely discontinued after 2 years of their marriage.
She spoke to her children about her situation and thought that they, particularly her older child, are able to understand her. She said that I explained to my children that "I am still your parent but my role will change." She stated that "I am an adult and experienced the life, marriage and raising my children. My insistence on surgery is not an impulsive decision. I really feel that I am a prisoner of my body and I am looking for freedom. I will certainly have surgery even if only one day of my life is remained. I am ready to endure any pain and discomfort so that I can live with my true identity and my name." in further evaluations we realized that there is no family history of psychiatric disorder, except for the gender identity disorder in her son. At counseling sessions, she and her husband mutually agreed to divorce. After divorce, she wore men's clothes and was very happy and satisfied. She thought that she is a male partner who can love a woman. She used to feel that her life is not worthy because She had the feeling that she is really a man who trapped in a wrong body. Mental status examination revealed no psychiatric disorder. She was hopeful, energetic and assertive. The results of the karyotype and sex hormone tests were within normal range and in line with the gender determined as a woman. MMPI revealed high scores in Mf scale that suggests serious doubts about traditional feminine roles. The BEM gender Role Inventory showed a higher normal masculinity and normal limit femininity scores.

\section{Results}

As far as we know, all reports of family aggregation of gender dysphoria, included twins or siblings, and concordance of transsexualism has not been reported in a mother and her son. This could be due to reduced fertility rates because of sex reassignment surgery in patients with gender dysphoria.

It has been suggested that there is low probability of marriage in male adolescents and youths with late-onset gender dysphoria. Female adolescents and youths with early-onset gender dysphoria almost always show sexual attraction towards females. Natal females with late-onset gender dysphoria usually have sexual attraction to males and perceive themselves as a homosexual man after SRS and becoming a man. Although case \#2 as a natal girl reported typical boyish behaviors in childhood which is consistent with an early-onset gender dysphoria, Her marriage to men can be explained with social and cultural expectations.. In fact, some families may believe that marriage can solve the gender role problems. They may think that after a marriage, a transgender will change in mind and behavior. Although Prescription of estrogen is a recommended clinical decision to accelerate the development of secondary sexual characteristics, it is not legally permissible in Iran. 


\section{Mental Health \& Human Resilience International Journal}

\section{Conclusions}

The social and cultural differences of a rural community in western Iran and the urban community can explain the early detection of gender diversity in a boy towards his mother. In the other hand, there is more cultural resistance against boy's girlish behaviors in comparison of girl's boyish behaviors. Gender dysphoria in pair of a mother and her son suggests that heritability might raise an etiologic contribution in addition to a shared identity hypothesis in this disorder. More researches are needed to define intermediate phenotypes and genetic and non-genetic components that contribute to development of gender dysphoria.

The concordant GD in an assigned adolescent male and his biological mother suggest the role of innate genetic factors and/or shared identity problem in development of GD. The occurrence of such a rare incidence could be explained by cultural, environmental and social factors affecting awareness of the varied forms of gender identity development.

\section{Acknowledgements}

The authors thank all the colleagues in Legal Medicine Council of Tehran.

\section{References}

1. American Psychiatric Association (2013) Diagnostic and Statistical Manual of Mental Disorders, Fifth Edition (DSM-5), American Psychiatric Association, Arlington, VA.

2. American Psychiatric Association (2000) Diagnostic and statistical manual of mental health disorders, $4^{\text {th }}$ (Edn.), Text Revision (DSM-IV-TR). American Psychiatric Association, Washington DC.

3. Arcelus J, Bouman WP, Van Den Noortgate W, Claes L, Witcomb G, et al. (2015) Systematic review and meta- analysis of prevalence studies in transsexualism. Eur Psychiatry 30(6): 807-815.

4. Bailey JM, Dunne MP, Martin NG (2000) Genetic and environmental influences on sexual orientation and its correlates in an Australian twin sample. J Personal Soc Psychol 78(3): 524-536.

5. Arcelus A, Cherkas L, Spector T, Rahman Q (2011) Genetic and environmental influences on female sexual orientation, childhood gender typicality and adult gender identity. PLOS ONE 6: e21982.

6. Heylens G, De Cuypere G, Zucker KJ, Schelfaut C, Elaut E, et al. (2012) Gender identity disorder in twins: A review of the case report literature. J Sex Med 9(3): 751-757.

7. Hwu HG, Yeh EK, Chang LY (1989) Prevalence of psychiatric disorders in Taiwan defined by the Chinese Diagnostic Interview Schedule. Acta Psychiatr Scand 79(2): 136-147.

8. Loehlin JC, Jonsson EG, Gustavsson JP, Stallings MC, Gillespie NA, et al. (2005) Psychological masculinityfemininity via the gender diagnosticity approach: heritability and consistency across ages and populations. J Personal 73(5): 1295-1319.

9. Zucker KJ, Bradley SJ, Owen-Anderson A, Kibblewhite SJ, Wood H, et al. (2012) Demographics, behavior problems, and psychosexual characteristics of adolescents with gender identity disorder or transvestic fetishism. Journal of Sex and Marital Therapy 38(2): 151-189.

10. Zucker KJ, Bradley SJ, Sanikhani M (1997) Sex Differences in Referral Rates of Children with Gender Identity Disorder: Some Hypotheses. Journal of Abnormal Child Psychology 25(3): 217-227.

11. Zucker KJ, Lawrence AA, Kreukels B (2016) Gender Dysphoria in Adults. Annual Review of Clinical Psychology 12: 217-247. 